\title{
Microfluidic Engineering
}

\author{
MEOENED \\ FA1!20 \\ OSTI
}

Phillip H. Paul

\section{Prepared by}

Sandia Nationai Laboratories

Albuquerque, New Mexico 87185 and Livermore, California 94550

Sandia is a multiprogram laboratory operated by Sandia Corporation, a Lockheed Martin Company, for the United States Department of Energy under Contract DE-AC04-94AL85000.

Approved for public release; further gissemination unlimited.

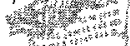
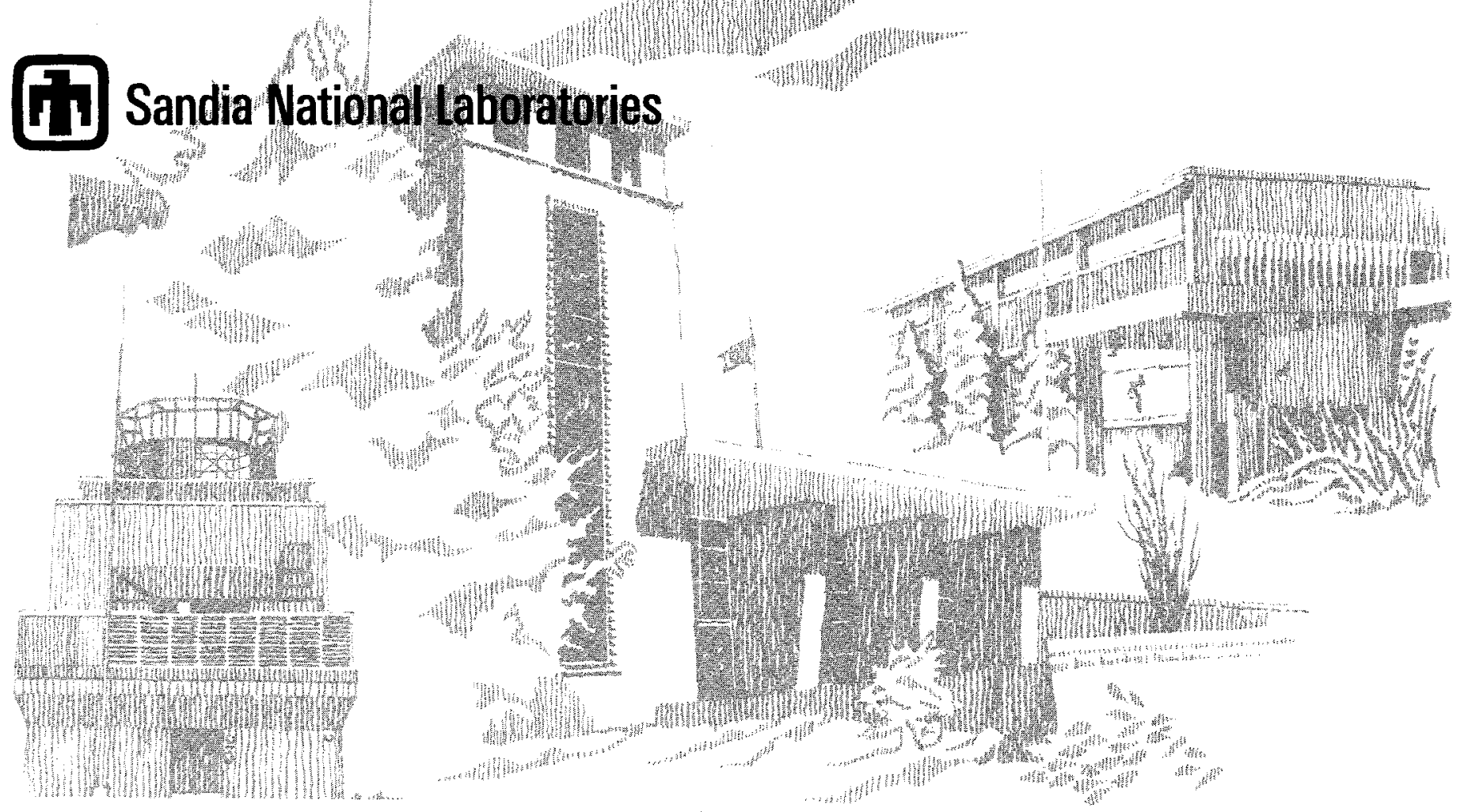
Issued by Sandia National Laboratories, operated for the United States Department of Energy by Sandia Corporation.

NOTICE: This report was prepared as an account of work sponsored by an agency of the United States Government. Neither the United States Government, nor any agency thereof, nor any of their employees, nor any of their contractors, subcontractors, or their employees, make any warranty, express or implied, or assume any legal liability or responsibility for the accuracy, completeness, or usefulness of any information, apparatus, product, or process disclosed, or represent that its use would not infringe privately owned rights. Reference herein to any specific commercial product, process, or service by trade name, trademark, manufacturer, or otherwise, does not necessarily constitute or imply its endorsement, recommendation, or favoring by the United States Government, any agency thereof, or any of their contractors or subcontractors. The views and opinions expressed herein do not necessarily state or reflect those of the United States Government, any agency thereof, or any of their contractors.

Printed in the United States of America. This report has been reproduced directly from the best available copy.

Available to DOE and DOE contractors from

Office of Scientific and Technical Information

P.O. Box 62

Oak Ridge, TN 37831

Prices available from (615) 576-8401, FTS 626-8401

Available to the public from

National Technical Information Service

U.S. Department of Commerce

5285 Port Royal Rd

Springfield, VA 22161

NTIS price codes

Printed copy: A03

Microfiche copy: A01

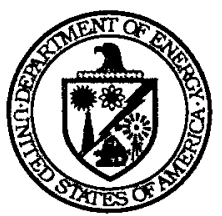




\section{DISCLAIMER}

Portions of this document may be illegible in electronic image products. Images are produced from the best available original document. 
SAND99 - 8212

Unlimited Release

Printed December 1998

\title{
Microfluidic Engineering
}

\author{
Phillip H. Paul \\ Sandia National Laboratories \\ Livermore CA 94550
}

\begin{abstract}
The ability to generate high pressures using electrokinetic pumping of liquid through porous media is reported. Pressures in excess of 8000 psi have been achieved using capillaries $(<100 \mu \mathrm{m}$ i.d.) packed with micron-size silica beads. A model is presented which accurately predicts absolute pressures, flowrates and power conversion efficiencies as well as the experimentally observed dependencies on pore size, applied electric field and electrical properties of the fluid. This phenomenon offers the possibility of creating a new class of microscale fluid devices, electrokinetic pumps and valves, where the performance improves with scale-down by taking advantage of microscale processes.
\end{abstract}


Acknowledgement The author gratefully acknowledges Dr.s Don Arnold, Dave Rakestraw, Stan Kravitz and Greg Frey, Mr. Ken Hencken and Mr. Mike Oborny for their contributions to and participation in this project and Dr. Tim Shepodd for supplying samples of porous polymers. Portions of this report are taken from the manuscript 'High pressure electrokinetic liquid micropumps,' P. H. Paul, D. W. Arnold, J. J. Scherer and D. J. Rakestraw (to be submitted to Science, 1998). This work was supported by Sandia National Laboratories, Laboratory Directed Research and Development program. 


\title{
Microfluidic Engineering
}

\author{
Phillip H. Paul 8351 \\ Sandia National Laboratories \\ Livermore CA 94550
}

Introduction It has long been recognized that electroosmotic flow is capable of transporting liquids through fine porous media. This basic property has been applied to technologies ranging from in situ cleansing of contaminated groundwater [1] to capillary electrochromatography [2]. Rastogi [3] reported on experiments where a voltage was applied across an aqueous electrolyte-filled glass frit mounted in a glass tube. He showed that electroosmotic flow can generate fluid pressure. However in his experiments, the maximum pressure generated was less than $0.75 \mathrm{psi}$ with the response becoming nonlinear for total applied voltages over $150 \mathrm{~V}$. In the current work we present experimental data that demonstrates the generation of pressures in excess of 8,000 psi using electroosmotic flow through a micro-porous medium. We find that the pressure generated scales linearly with the applied voltage and inversely with the square of the pore diameter. We present a model for electrokinetic flow in a porous medium that yields relationships for flowrate, pressure, current draw and power conversion efficiency. We also report the results of an experimental parametric study performed using silica bead-packed capillaries as the porous medium that shows excellent agreement with the model.

Theory The theory of electrokinetic flow in an open capillary is given by Rice and Whitehead [4]. The electrochemical double layer formed at the interface between the liquid and solid is described by two parameters: the thickness of the diffuse layer or Debye length, $\mathrm{K}^{-1}$; and, the 'zeta' potential, $\psi_{\mathrm{o}}$, being the electrostatic potential at the plan of shear or the potential between the Stern and diffuse components of the double layer. The actual value of $\psi_{0}$ depends on the nature of the solid material, temperature, the $\mathrm{pH}$ and the type and concentration of the ions in the electrolyte [5]. The net charge density resulting from the presence of the double layer can be related to a potential field, $\psi$, via Poisson's equation

$$
\varepsilon \nabla^{2} \psi=-\rho_{e}
$$

where $\varepsilon$ is the permittivity of the fluid. For steady creeping or 'Stokes' flow the momentum equation may be written as

$$
\eta \nabla^{2} \vec{u}=-\nabla P-\rho_{e} \vec{E}
$$


Here $\eta$ is the dynamic viscosity of the fluid, $u$ is the velocity, $\mathrm{P}$ is the pressure, and $\mathrm{E}$ is an externally applied electric field. Equations 1 and 2 are subject to boundary conditions of axial symmetry, $\psi(R)=\psi_{0}$ where $R$ is the radius of the capillary and no-slip at the wall (i.e. $u(R)=0$ ).

Combining equations 1 and 2 , the velocity profile is given by

$$
u(r)=\frac{-\nabla P R^{2}}{4 \eta}\left(1-r^{2} / R^{2}\right)-\frac{\varepsilon E \psi_{o}}{\eta}\left(1-\psi(r) / \psi_{o}\right)
$$

For a double layer that is thin compared to the radius of the channel (i.e. $k \mathrm{R}$ large), $\psi$ is non-zero only in the immediate vicinity of the wall, hence the electrokinetic term (second term on the right of equation 3) is zero at the wall but within several Debye thicknesses rises to a constant value which is maintained over the remainder of the fluid. The velocity profile appears as a linear superposition of parabolic pressure-driven pipe flow and pluglike electrokinetic flow. The average fluid velocity, $u_{m}$, is obtained by integrating the velocity profile over the area of the capillary

$$
u_{m}=\frac{-\nabla P R^{2}}{8 \eta}-\frac{\varepsilon E \psi_{o}}{\eta}(1-\xi)
$$

Here $\xi$ is the area-average of $\psi / \psi_{0}$, a dimensionless function of $\kappa R$ and $\psi_{0}$ which accounts for the overlap of the double layers from opposing walls [6].

The maximum net electrokinetic flow occurs in the absence of any opposing axial pressure gradient and is given by

$$
u_{o} \equiv u_{m}(\nabla P=0)=\frac{-\varepsilon E \psi_{o}}{\eta}(1-\xi)
$$

Note that this velocity is independent of the capillary diameter and is proportional to the electric field. The value of an axial pressure gradient, $\nabla \mathrm{P}_{\max }$, applied so as to oppose the electrokinetic flow and sufficient to achieve a condition of zero net flow is given by

$$
\nabla P_{\max } \equiv \nabla P\left(u_{m}=0\right)=\frac{-8 \varepsilon E \psi_{o}}{R^{2}}(1-\xi)
$$

The condition of zero net flow is achieved when the Lorentz force on the ions in the diffuse layer due to the applied electric field is balanced by that on the whole fluid due to the opposing pressure gradient. In this case, according to equation 3 , the velocity profile exhibits a positive electrokinetic flow along the walls which is balanced by a negative parabolic (pressure-driven) flow back down the center of the channel. Thus electrokinetic flow into a closed volume will transport fluid to the closed end to create an opposing differential pressure across the capillary which in steady state results in a condition of zero net flow. 
Experimental investigation of electrokinetic flow in the limit of small capillary diameter is hampered by the difficulty of fabricating such capillaries and by the low volumetric flowrates produced. Flow through a larger diameter channel containing a micro-porous medium offers a practical alternative. Porous material can be viewed as an array of intertwined fine capillaries (here termed 'tubules') providing a means to achieve small effective channel diameters. At the same time the cross sectional area of the porous medium can be large enough to prove sufficient flow for measurements and ultimately useful work. In the tubule model the porous medium is describes by two parameters: the porosity, $\varphi$, being the wetted volume fraction; and the tortuosity, $\gamma$, being the length of the tubules per unit length of the medium. For packed spherical beads the effective diameter of the tubules, $d_{t}$, is directly proportional to the bead diameter, $d_{B}$, and can be estimated given the porosity of the medium (i.e. $d_{t}=f(\varphi) d_{B}[7]$ ).

Consider then a section of macroscopic fused silica capillary of length $L$ and diameter $D$ (cross sectional area A) which is packed with fused silica beads of diameter $d_{B}<<D$. A DC voltage is applied across the system to yield a voltage $V$ across the packed section. The electric field inside of the tubules is reduced by the tortuosity. The total electrokinetic flow rate without any applied back pressure is $\mathrm{Q}_{0}=\mathrm{AU}_{0}$ which is proportional to the mean velocity in the tubules as further reduced by the porosity and the tortuosity. Thus the apparent velocity is

$$
U_{o}=u_{o} \frac{\varphi}{\gamma^{2}}
$$

Using equation 6 we can also state, the maximum pressure difference produced across the packed section for a condition of zero net flow is

$$
\Delta P_{\max }=\frac{-32 \varepsilon \psi_{o}}{d_{B}^{2} f^{2}(\varphi)} V(1-\xi)
$$

This result predicts that electroosmotic flow in a micro-porous medium should be capable of generating very high pressures that will scale linearly with the applied voltage and inversely with the square of the bead diameter. The experimental data that follows validates these relationships.

Results and Discussion The basic experimental apparatus consisted of a section of fused silica capillary which was packed with silica beads. The upstream end of the capillary was placed in a reservoir which was open to ambient pressure and which contained a platinum wire electrode. The downstream end of the capillary was fit into a plastic HPLC ' $T$ ' with the side leg equipped with a platinum wire electrode. The third leg of the ' $T$ ' was used as a port to attach various diagnostics. Two methods were used for pressure measurements: A close-coupled miniature pressure transducer which is a flow through design and thus allowed the transducer body to be purged of any air. The second method, which was our initial approach, determined pressure by measuring the compression length of a column of air trapped in a long section of open capillary which was sealed at the far end. The results of both methods were consistent to within the 
accuracy and reproducibility of knowing that the flow was fully stopped and measuring the compression length in the second method.

Experiments were performed with capillaries packed with both coated $\left(\mathrm{C}_{18} \mathrm{H}_{37} \mathrm{Si}\left(\mathrm{CH}_{3}\right)_{2}\right.$, ODS) and uncoated non-porous silica (NPS) beads [8]. Two electrolyte solutions were employed: an 80:20 mixture of acetonitrile and water buffered with $4 \mathrm{mM}$ aqueous sodium tetraborate; and pure water buffered with 1 or $2 \mathrm{mM}$ borate. For both solutions the Debye thickness was calculated to be in the range of 5 to $6 \mathrm{~nm}$. Based on conductivity measurements we find porosities around $\varphi \approx 0.32$ which gives the effective tubule diameter between one-forth and one-fifth of the bead diameter [7]. The smallest beads used in the present study are $0.5 \mu \mathrm{m}$ in diameter thus the ratio of the tubule radius to the Debye length is of order $\mathrm{kR}>10$ and as such the correction for double layer overlap is a secondary effect (i.e. $0 \leq \xi \leq 0.2$ ).

The first set of pressure measurements were made using 50,75 and $100 \mu \mathrm{m}$ i.d. capillaries packed to various lengths with $3 \mu \mathrm{m}$ diameter ODS coated NPS beads using the 80:20 ACN:water electrolyte. The results are shown in figure 1 where we find that the total pressure produced is linearly proportional to the total voltage applied across the packed section and is independent of the diameter of the capillary. To investigate the effect of particle diameter on the pressure generated, experiments were conducted using $1.5 \mu \mathrm{m}$ ODS coated NPS beads and $4.5 \mu \mathrm{m}$ NPS beads again with the 80:20 ACN:water electrolyte and $0.5,0.6$ and $5 \mu \mathrm{m}$ NPS beads and $1.5 \mu \mathrm{m}$ ODS coated NPS beads with the water electrolyte. These results, along with the $3 \mu \mathrm{m}$ bead data, are shown in figure 2 plotted as differential pressure generated per applied volt as a function of bead diameter. All of the data shown in figure 2 are found to be consistent with the theoretical result. The consistency of the data in figure 2 also implies that the zeta potential on the packing is near equal for conditions of ODS coated NPS beads and NPS beads in ACN:water buffered with $4 \mathrm{mM}$ borate and for NPS beads in water buffered with $1 \mathrm{mM}$ borate, respectively.

The combination of small bead hence small tubule diameter and long capillaries can be used to generate large absolute pressures. Tests performed using a $10 \mathrm{~cm}$ section of $50 \mu \mathrm{m}$ i.d. (350 $\mu \mathrm{m}$ o.d.) capillary packed with $0.6 \mu \mathrm{m}$ NPS beads gave $6000 \mathrm{psi}$ at an applied voltage of $1 \mathrm{kV}$ and at a total current draw of a few $\mu \mathrm{A}$. Joule heating under these conditions is well below the point at which non-ideal behavior (e.g. boiling of the fluid) would set in. However, upon increasing the voltage, it was only possible to obtain pressures of 8000 to 9000 psi before the Teflon-clad capillary blew out of the HPLC fittings (at a force of nominally $5 \mathrm{~N}$ on the end of the capillary). The highest pressures observed in the experiments were thus limited by the mechanics of the apparatus and the ability to measure high pressures rather than any fundamental physical limit to the generation of electrokinetic flow.

The mean flow velocity $U_{o}$ was measure by fitting the diagnostic port with a long section of open capillary (as initially filled with air) and visually measuring the velocity of the liquid-air interface. The ratio of mean velocity to the applied field across the packed section, as measured for flow into an open system is shown in figure 3. The results are consistent with the predictions of equation 7 , that the flow velocity is independent of bead diameter and proportional to the field applied across the packed section. Knox and Grant 
[2] measured the electrokinetic flow velocity through both slurry- and drawn-packed capillaries. Their results show a linear dependence on applied electric field and they conclude that the electroosmotic velocity is unaffected by particle size for bead diameters between 1.5 to $50 \mu \mathrm{m}$. The weak dependence of mean flow velocity on bead diameter observed here suggests that the ratio $\varphi / \gamma^{2}$ (see equation 7) is also relatively independent of bead diameter. The porosities, as determined from the measured conductivity ratios [7], also fall into a relatively narrow range suggesting that the tortuosities of the packed columns are also relatively independent of bead diameter.

The net electrokinetically-driven flow rate through the porous medium as subject to a back pressure, $\Delta \mathrm{P}$, can be written using equation 4

$$
Q=U_{o} A\left(1-\Delta P / \Delta P_{\text {max }}\right)
$$

For a given packed capillary both $\mathrm{U}_{\mathrm{o}}$ and $\Delta \mathrm{P}_{\max }$ are proportional to the applied voltage, thus for a fixed back pressure the flowrate is predicted to scale linearly with the applied voltage. To test this result, a section of bead-packed capillary was connected to the diagnostics port using the flow-through pressure transducer as the coupling. A voltage was applied across the upstream capillary. The downstream capillary provides a pressure drop to ambient and also acts as a flowmeter through the measured pressure [9]. Measurements performed using capillaries packed with the 1.5 and $3 \mu \mathrm{m}$ ODS coated NPS beads, showed a linear relation between the measured pressure, hence flowrate, and the applied voltage. These experiments were conducted using downstream capillaries of different diameters, lengths and packed with different bead diameters to proved an adjustable back pressure. For a subset of these cases, the total flowrate was measured using the moving meniscus method described above. Figure 4 shows a flow.vs.pressure curve as measured for an electrokinetically driven capillary operated at a fixed voltage and packed with $1.5 \mu \mathrm{m}$ ODS coated NPS beads. The results are in good agreement with the theoretical prediction of equation 9 (also shown plotted in figure 4).

The rate of work done by a pump and imparted to the fluid as available hydraulic power is the product of the differential pressure generated and the volumetric flow rate. Applying equation 9 the rate of work available has a maximum at $W_{\max }=U_{o} A \Delta P_{\max } / 2$. For a 100 $\mathrm{mm}$ long $50 \mu \mathrm{m}$ i.d. capillary packed with $0.6 \mu \mathrm{m}$ NPS beads using an electrolyte of water buffered with $1 \mathrm{mM}$ borate we measure a zero-load flow rate of $\mathrm{Q}_{\mathrm{o}}=0.008 \mu \mathrm{L} / \mathrm{min}$ and $\Delta \mathrm{P}_{\max }=6000 \mathrm{psi}$ at an electrical power input of $94 \mu \mathrm{W}$, this gives a conversion efficiency of $2.9 \%$. To understand the efficiency of the device it is useful to separate the current into two components: a conduction current due to the finite conductivity of the electrolyte; and a convection current as given by the product of the net charge density with the velocity as integrated over the area of the capillary [4]. This convection current is inherent in electroosmotic flow and is a direct result of fluid motion driven by the Lorentz force on the net charge in the fluid. The conduction current represents a purely resistive loss which is incurred by the need to add ions to the fluid to stabilize the electrolyte and to control the thickness of the double layer. The conductivity of the electrolytes used here was measured in the range of $0.01 \mathrm{mho} / \mathrm{m}$ in which case the observed currents are almost entirely due to conduction. Calculations suggest that the ion concentration could be reduced by an order of 
magnitude without any significant reduction in the electroosmotic flow- or pressuregenerating performance of the devices considered here. This would yield a proportional decrease in the conduction current and thus a substantial increase in the power conversion efficiency.

Figure 5 shows the performance of several different classes of liquid micropumps as described in the literature. These include electrohydrodynamic pumps $[10,11]$, diaphragm pumps using check valves $[12,13,14]$ and diffusers [15], and the variety of micropumps reviewed by Shoji and Esashi [16]. The diaphragm pump using a check valve reported by Woias et al. [13] displays a power conversion efficiency which appears to be $2.5 \%$. For diaphragm pumps using diffusers, power conversion efficiencies are of the order $0.15 \%$. Figure 5 also shows results for three electrokinetically driven bead-packed capillaries. The diaphragm, peristaltic and electrohydrodynamic pumps considered tend to produce high flow rates but only at modest pressures. In contrast electrokinetic pumping is distinct in producing very high pressures at a modest flow rate.

Current and Power Conversion Efficiency As noted above, the current drawn is the sum of ohmic conduction and charge convection components. For a univalent electrolyte the current may be written

$$
I=E \int\left(v_{+} n_{+}(r)+v_{-} n_{-}(r)\right) d A+\int \rho_{e}(r) u(r) d A
$$

Here the $v_{j}$ are the mobilities of the positive and negative ions in the electrolyte. Using equations 1,3 and 6 above, and following several integrations by parts, this can be rewritten as

$$
I=E A\left[\sigma_{o} \xi_{3}+\left(\varepsilon^{2} \psi_{o}^{2} / R^{2} \eta\right)\left(\xi_{2}-8(1-\xi)^{2} \Delta P / \Delta P_{m}\right)\right]
$$

Here $\sigma_{o}=n_{o}\left(v_{+}+v_{-}\right)$is the electrical conductivity of the bulk fluid,

$$
\begin{gathered}
\xi \equiv \frac{2}{R^{2} \psi_{o}} \int_{0}^{R} \psi r d r \\
\xi_{2} \equiv \frac{2}{\psi_{0}^{2}} \int_{0}^{R}\left(\frac{\partial \psi}{\partial r}\right)^{2} r d r
\end{gathered}
$$

and, invoking the Boltzmann description to relate the local charge densities to the field $\psi$,

$$
\xi_{3} \equiv \frac{2}{R^{2}} \int_{0}^{R} \cosh \left(\frac{e \psi}{k_{B} T}\right) r d r+\frac{v_{-}-v_{+}}{v_{-}+v_{+}} \frac{2}{R^{2}} \int_{0}^{R} \sinh \left(\frac{e \psi}{k_{B} T}\right) r d r
$$

This may be integrated by parts to yield 


$$
\xi_{3}=\cosh \left(\frac{e \psi_{o}}{k_{B} T}\right)-\left.\frac{1}{2} \frac{e}{k_{B} T}\left(\frac{d \psi}{d \kappa r}\right)^{2}\right|_{\kappa R}+\left.\frac{v_{-}-v_{+}}{v_{-}+v_{+}} \frac{2}{\kappa R} \frac{e}{k_{B} T}\left(\frac{d \psi}{d \kappa r}\right)\right|_{\kappa R}
$$

The function $\xi_{3}$ gives the change in the ohmic conductivity resulting from the excess charge density in the double layers. Note that coefficient multiplying the second term in equation 3 can take either sign depending on the relative values of the mobilities.

To write an expression for power conversion efficiency it is first necessary to consider the magnitude of the rate of ' $p d v$ ' work done in compressing the fluid,

$$
\dot{W}_{p d v}=Q \Delta P\left[\frac{b_{T}}{2}\left(P_{f}+P_{i}\right)\left(1-\frac{3 b_{T}}{2} \frac{P_{f}^{3}-P_{i}^{3}}{P_{f}^{2}-P_{i}^{2}}\right)\right]
$$

Here the subscripts refer to the initial and final pressure of the fluid and $b_{\mathrm{T}}$ is the isothermal compressibility of the fluid (of order $5 \times 10^{-5} / \mathrm{atm}$ for water). For the extreme case of pumping water from one to $500 \mathrm{~atm}$, the second order term in equation 14 is of order 0.015 and is thus negligible in favor of the first order term. Further, for the case considered, the first order term is a similarly small fraction of $\mathrm{Q} \Delta \mathrm{P}$.

The power conversion efficiency (i.e. the hydraulic power available as flowrate - pressure difference product per unit total electrical power applied) to first order in the compressibility is then given by

$$
E f f=\frac{Q \Delta P\left(1-b_{T}\left(P_{f}+{ }_{i}\right) / 2\right)}{I V}
$$

Substituting the expressions for flowrate and current, using equation 9 above and defining $\cdot \mathrm{y} \equiv \Delta \mathrm{P} / \Delta \mathrm{P}_{\mathrm{m}}$ yields

$$
E f f=\frac{8(1-\xi)^{2} y(1-y)\left(1-b_{T}\left(P_{f}+P_{i}\right) / 2\right)}{(\kappa R)^{2}\left(\frac{k_{B} T}{e \psi_{o}}\right)^{2} \beta \xi_{3}+\xi_{2}-8 y(1-\xi)^{2}}
$$

where $\beta=\eta\left(v_{+}+v_{-}\right) / 2 \varepsilon k_{B} T$. The three terms in the denominator correspond to power consumed by joule heating, forward charge convection by the electric field and reverse charge convection by the pressure gradient. The factor containing $\xi$ in the numerator introduces the reduction of flowrate and pressure due to overlap of the double layers. Whereas the term containing $b_{\mathrm{T}}$ reflects the loss due to finite compressibility of the liquid. Note, the expression for the efficiency as written is independent of the voltage applied across the device. Rather the operating voltage is hidden in parameter $y$ which defines the pressure load operating condition as a fraction of the maximum pressure $\Delta \mathrm{P}_{\mathrm{m}}$ which contains a linear dependence on the voltage. In the ideal case (i.e. a nonconducting, incompressible liquid hence $\beta=0$ and $b_{\mathrm{T}}=0$ ) the efficiency approaches $100 \%$ in the limit as $\kappa \mathrm{R}$ goes to unity in which limit $(1-\xi)$ goes to zero. In effect, the perfect electrokinetic 
pump can approach 100\% power conversion efficiency in the limit that the rate of work available as hydraulic power in the fluid goes to zero.

It is important to note that all of the expressions given above are derived without invoking a solution to the Poisson-Boltzmann (PB) equation. The PB equation must in fact be solved to evaluate the $\xi$ integrals. Equations 10 and 13 are specialized to the case of a univalent electrolyte but may be generalized to a non-univalent electrolyte by replacing the mobilities with $z_{j}^{2} v_{j}$ (where the $z_{j}$ are the ion charge numbers) and by writing the ion number densities as $n_{j}=n_{o} \exp \left(-z_{j} e \psi / k_{B} T\right)$.

For a univalent electrolyte and in the Debye-Huckel limit [see ref. 6] the $\xi(x \equiv \kappa R)$ integrals become

$$
\begin{gathered}
\xi=2 I_{1}(x) / x I_{o}(x) \\
\xi_{2}=x^{2}\left(\left(I_{1}(x) / I_{o}(x)\right)^{2}+2 I_{1}(x) / x I_{o}(x)-1\right) \\
\xi_{3}=1+\frac{v_{-}-v_{+}}{v_{-}+v_{+}} \frac{e \psi_{o}}{k_{B} T} \xi
\end{gathered}
$$

Here $I_{0}$ and $I_{1}$ are modified Bessel functions. The later relation is only valid for $e \psi_{0} / k_{B} T<<$ 1. In the literature the 'Debye-Huckel' assumption is most often taken to imply $\xi_{3}=1$. The value of the $\xi$ integral is reasonably well represented by the Debye-Huckel solution for all $\kappa R>2$ and $e \psi_{o} / k_{B} T<4$. However this is not the case for $\xi_{2}$ and $\xi_{3}$, rather a full solution to the $\mathrm{PB}$ equation is required to properly evaluate these functions for $\mathrm{e} \psi_{\mathrm{o}} / \mathrm{k}_{\mathrm{B}} \mathrm{T}$ greater than of order $1 / 2$.

To generalize these expression to a porous medium: The current is given by

$$
I=E A\left[\sigma_{e f f} \xi_{3}+\frac{\varphi}{\gamma^{2}}\left(\varepsilon^{2} \psi_{o}^{2} / R^{2} \eta\right)\left(\xi_{2}-8(1-\xi)^{2} \Delta P / \Delta P_{m}\right)\right]
$$

Here $\sigma_{\text {eff }}$ is defined in ref. [7] and may be replaced by writing $\sigma_{\text {eff }}=\sigma_{o}(1+s) \varphi / \gamma^{2}$ where $\mathrm{s}$ is a higher function of porosity and possibly conductivity. The efficiency, for a porous material, may then be written

$$
E f f=\frac{8(1-\xi)^{2} y(1-y)\left(1-b_{T}\left(P_{f}+P_{i}\right) / 2\right)}{(\kappa R)^{2}\left(\frac{k_{B} T}{e \psi_{o}}\right)^{2} \beta(1+s) \xi_{3}+\xi_{2}-8 y(1-\xi)^{2}}
$$

In equations 18 and $19, \mathrm{R}$ is the radius of the tubules. For a bed of spherical beads the tubule radius is $R_{t}=f(\varphi) d_{B} / 2$ [ see ref. 7 ]. Since the value of $s$ is in general small compared to unity, the efficiency of a porous system is only slightly reduced from that of an open capillary having the same internal diameter as the tubules. 
Streaming potential and current The application of a pressure, $\Delta \mathrm{P}$, across an open or porous media filled system results in charge convection that can be measured either as a 'streaming potential,' $\Delta \mathrm{V}_{\mathrm{s}}$ (the voltage created across the system), or as a 'streaming current,' $I_{s}$. Using the result of equation 18 ,

$$
\begin{gathered}
\frac{I_{s}}{\Delta P}=\frac{A}{L} \frac{\varphi}{\gamma^{2}} \frac{\varepsilon \psi_{o}}{\eta}(1-\xi) \\
\frac{\Delta V_{s}}{\Delta P}=\left[\frac{\sigma_{o} \eta}{\varepsilon \psi_{o}}(1+s) \frac{\xi_{3}}{1-\xi}+\frac{\varepsilon \psi_{o}}{R^{2}} \frac{\xi_{2}}{1-\xi}\right]^{-1}
\end{gathered}
$$

These, combined with conductivity measurements, provide a means to obtain additional information about the parameters governing the system.

Materials Electrokinetic pumping studies were conducted with a variety of electrolytes (both aqueous and organic liquids) and a variety of porous materials. Bulk porous materials like porous ceramics or porous glass (as made using the Vycor process) provide a means to shape the electrokinetic pump to address particular applications. Bulk porous polymers can be cast in place and in any shape. Bulk porous materials can be produced with a wide range of pore diameter and also a higher porosity than possible with bead-packing. The obvious preference in an electrolyte is to achieve a very low bulk conductivity, hence lower current thus lower electrolytic gas generation rate and higher efficiency. However any reduction in conductivity must be balanced against the chemical stability of the electrolyte and must be compatible with the necessary electrode chemistry and charge transfer from the electrode to the porous material.

The possibility of using porous silicon (as produced by anodic etching) as a pump medium was investigated. The electrical conductivity of silicon (that is suitable for processing to porous silicon) is to large to use as a pump material. Protective layers of oxide deposited on the silicon are not sufficiently resistant to electrochemical attack by the electrolyte. Thus samples of fully oxidized porous silicon were produced for testing. Only relatively thin samples (e.g. sections of thinned wafers) could be manufactured and these were subject to a good deal of internal stress following oxidation. Owing to the thinness and fragility of the resulting material, porous silicon does not appear to be a good candidate for high pressure pumps. However fully oxidized porous silicon may find application as an EK pump host material for low pressure applications in wafer-scale systems.

Conclusion The demonstrated capacity of electroosmotic flow to convert electrical power to high specific force in a compact and efficient manner offers the opportunity to develop a new class of microscale actuators and drives which may have wide applicability to micro-electro-mechanical systems. Electrokinetic pumping requires no moving parts and takes advantage of microscale phenomenon which are well predicted by the tubular pore model presented in this report. Future efforts are planed to verify the model predictions regarding the effects of double layer overlap, to investigate the applicability of other types of micro-porous materials and electrolytes, and to develop the technology to fabricate electrokinetic micropumps on a wafer as a component of a micro fluidic system. 


\section{References}

1. A. P. Shapiro, P. C. Renaud and R. F. Probsein, PhysicoChemical Hydrodynamics $11,785-802$ (1989).

2. J. H. Knox and I. H. Grant, Chromatographia 32, 317-328 (1991).

3. R. P. Rastogi, J. Sci. and Industrial Research 28, 284-292, (1969)

4. C. L. Rice and R. J. Whitehead, J. Phys. Chem. 69, 4017-4024 (1965).

5. P. J. Scales, F. Grieser, T. W. Healy, L. R. White and D. Y. C. Chan, Langmuir 8, 965-974 (1992).

6 For a univalent electrolyte the charge density is given by $\rho_{e}=-2 n_{o} e \sinh \left(e \psi / k_{B} T\right)$ where $\mathrm{n}_{\mathrm{o}}$ is the concentration of the ions in the solution. The Debye length may then be written $\kappa^{-2}=2 n_{\circ} e^{2} / \varepsilon k_{B} T$. In the Debye-Huckel limit, specifically that the quantity $e \psi_{\mathrm{o}} / \mathrm{k}_{\mathrm{B}} \mathrm{T}$ is less than or of order unity, the charge density is approximated by $\rho_{e} \cong-\kappa^{2} e \psi / \varepsilon k_{B} T$ and the potential becomes $\psi(r)=\psi_{o} I_{0}(\kappa r) / I_{0}(\kappa R)$.

7. $f(\varphi)=\left(8 / 9 K_{c}\right)^{1 / 2} \varphi /(1-\varphi)$ R. F. Probstein, Physicochemical Hydrodynamics, (Wiley, NY, 1994) gives the constant as experimentally observed in the range $2.5<$ $\mathrm{K}_{\mathrm{c}}<5$. A. G. van der Put and B. H. Bijsterbosch, J. Colloid and Interface Sci. 75, 512-524 (1980) review methods to measure the porosity in terms of apparent conductivity of the fluid filled porous material, $\sigma_{\text {eff }}$ taken with respect to the electrical conductivity of the pure fluid, $\sigma$. Here we use the relation, $\sigma / \sigma_{e f f}=2 / \varphi(1+\varphi)$, that was proposed by J. G. Berryman, Phys. Rev. B27, 77897792 (1983), as appropriate to a condition of high bulk conductivity.

8. The coated particles are employed in our laboratory for conducting reverse phase electrokinetic chromatography are were used due to their availability. The coating is expected to slightly modify the zeta potential but otherwise is not expected to significantly alter the results presented in this paper.

9. The pressure driven flowrate through a porous medium of thickness $\mathrm{L}$ and total area A is given by Darcy's law as $Q=k_{D} A \Delta P / \eta L$ where $\mathrm{k}_{\mathrm{D}}$ is the Darcy permeability

10. A. Richter, A. Plettner, K. A. Hofmann and H. Sandmaier, Sensors and Actuators A29, 159-169 (1991).

11. C. C. Wong, D. R. Adkins, D. Chu, 'Development of a micropump for microelectronic cooling,' in proc. Microelectronics Systems (MEMS), (ASME, NY, 1996) DSC-Vol. 59, pp. 239-244.

12. M. Stehr, S. Messner, H. Sandmaier and R. Zengerle, Sensors and Actuators A 57, 153-157 (1996). M. C. Carozza, N. Croce, B. Magnani and P. Dario, J. Microeng. 5, 177-179 (1995). 
13. P Woias, R. Linnemann, R. Richter, A. Leistner and B. Hillerich, in Micro Total Analysis Systems, '98, D. J. Harrison and A. van den Berg ed.s (Kluwer Academic, London, 1998), pp. 383-385

14. S. Bohm, M. Dierselhuis, W. Olthuis and P. Bergveld, ibid pp. 391-394. O. Krusemark, A. Feustel and J. Muller, ibid pp. 399-402.

15. A. Olsson, G. Stemme and E. Stemme, Sensors and Actuators A46, 549-556 (1995). A. Olsson, P. Enoksson, G. Stemme and E. Stemme, J. Microelectromechanical Sys. 6, 161-166 (1997). T. Gerlach and H. Wurmus, Sensors and Actuators A50, 135-140 (1995). M. Koch, A. G. R. Evans and A. Brunnschweiller, J. Micromech. Microeng. 8, 119-122 (1998).

16. S. Shoji and M. Esashi, J. Microeng. and Micromechanics 4, 157-171 (1994). 


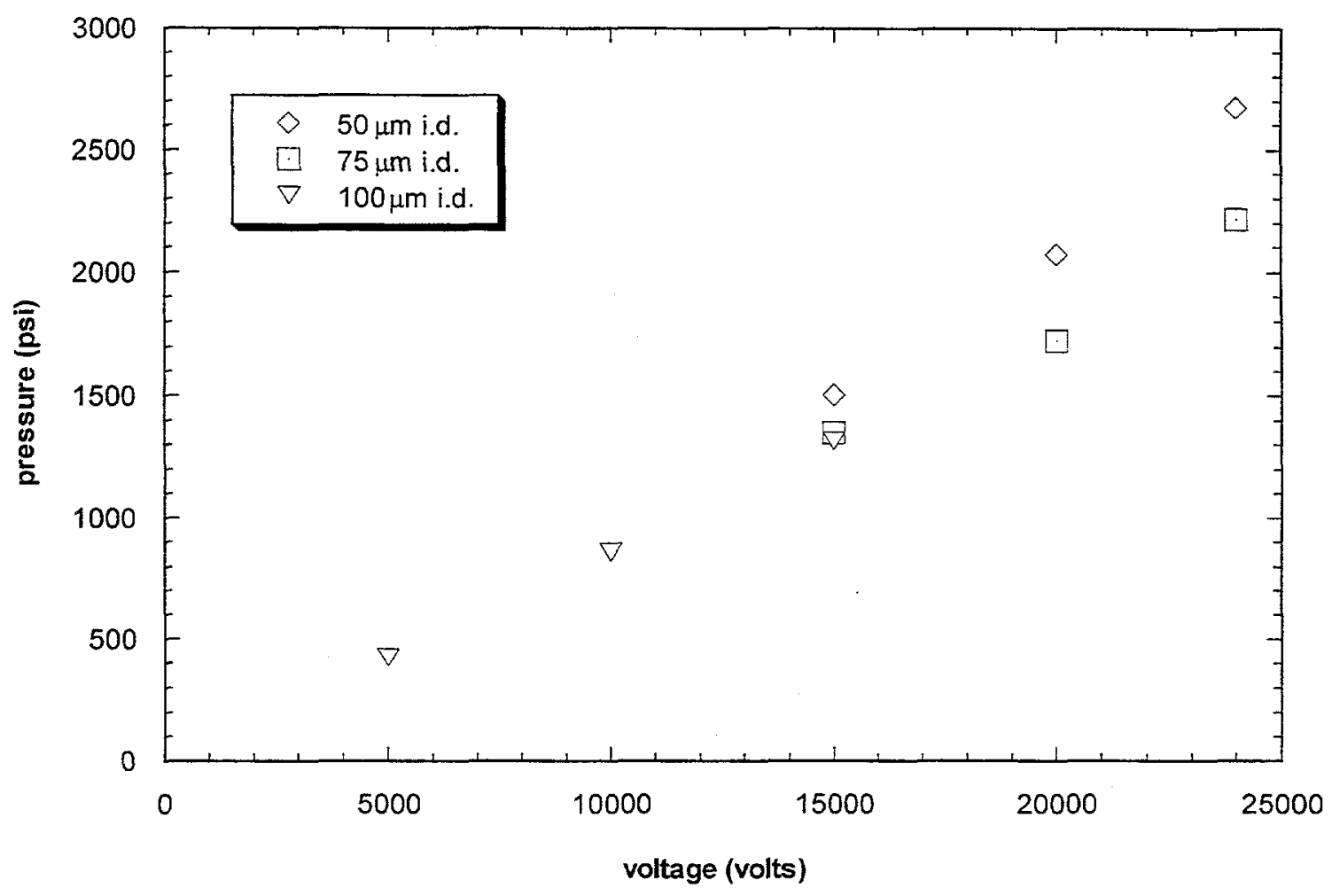

Figure 1. Maximum pressures generated as a function of applied voltage for capillaries of different internal diameters and lengths. Using packings of $3 \mu \mathrm{m}$ ODS coated NPS beads, 80:20 ACN:water with $4 \mathrm{mM}$ borate aqueous. 


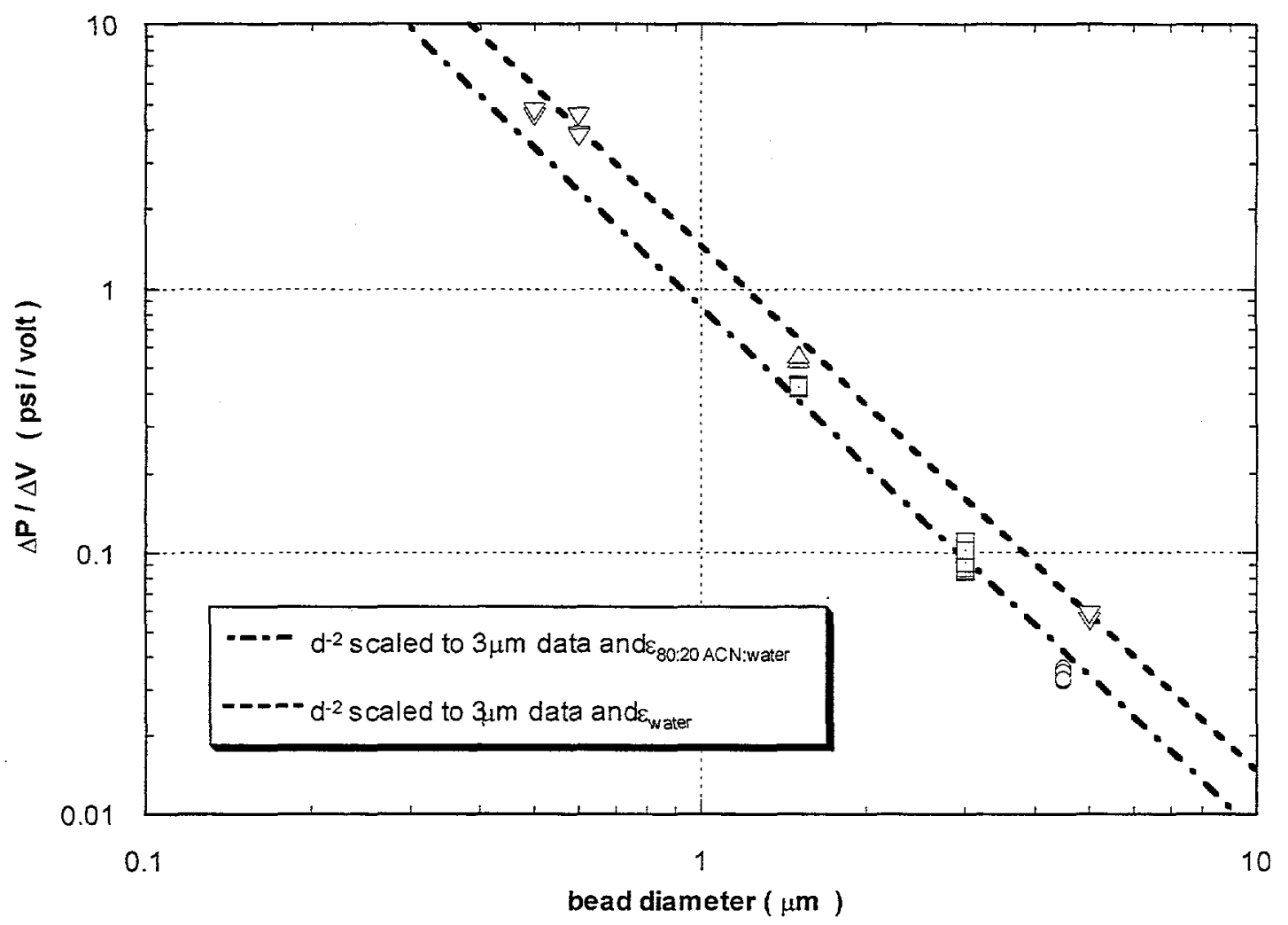

Figure 2. Pressure generated per applied volt plotted as a function of bead diameter. The two curves plot the inverse of the bead diameter squared as scaled to the mean of the data for the $3 \mu \mathrm{m}$ ODS coated NPS beads and for the permittivity of the solvents. $\square$ NPS and O ODS coated NPS in 80:20 ACN:water with $4 \mathrm{mM}$ borate aqueous and, $\triangle$ ODS coated NPS in aqueous $2 \mathrm{mM}$ borate, $\nabla$ NPS in aqueous 1 and $2 \mathrm{mM}$ borate. 


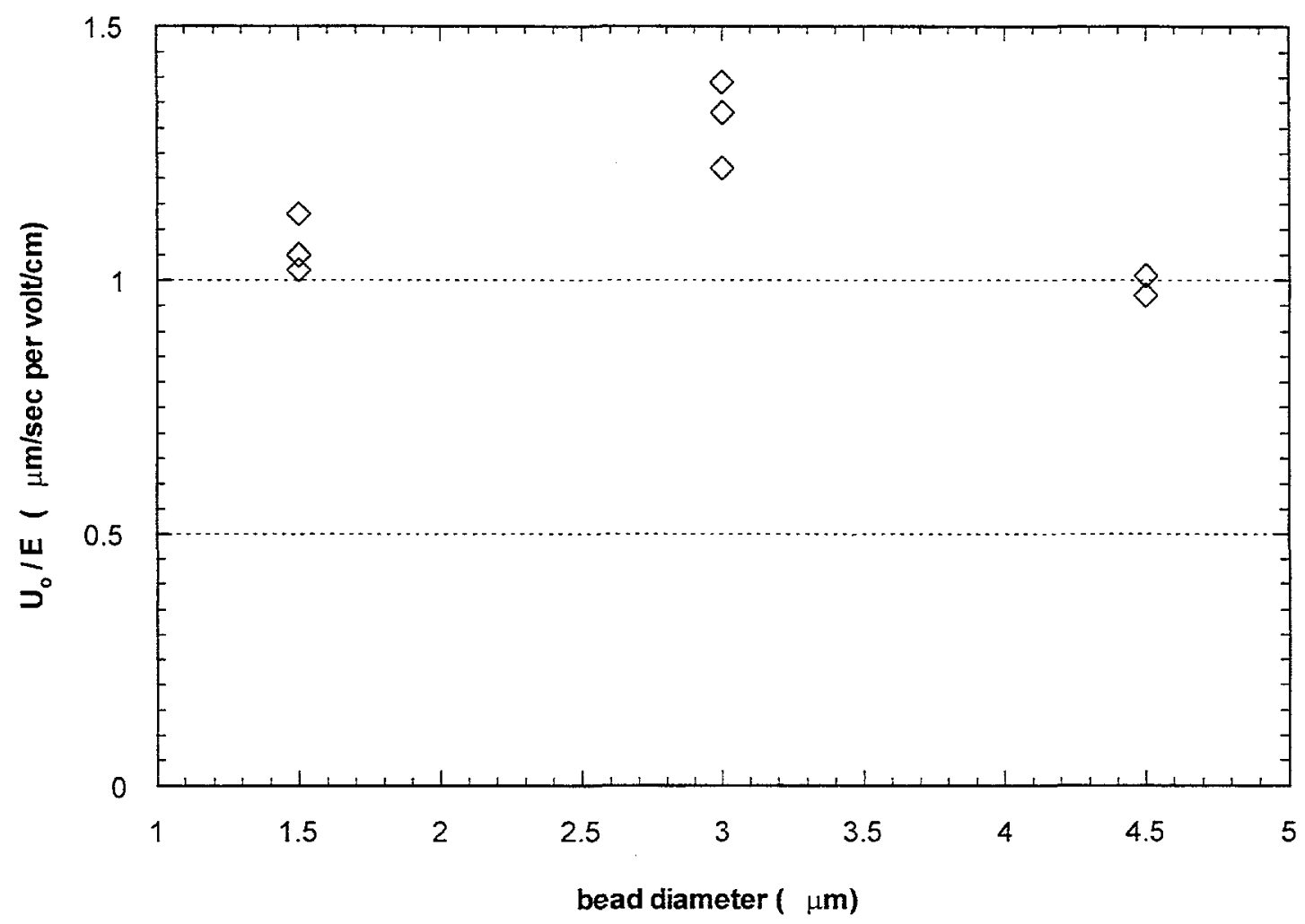

Figure 3. Measured mean flow velocity per unit applied electric field for conditions of no applied back-pressure plotted as a function of bead diameter. Results are shown for applied fields of 70,145 and $200 \mathrm{~V} / \mathrm{cm}$ at each bead diameter. 


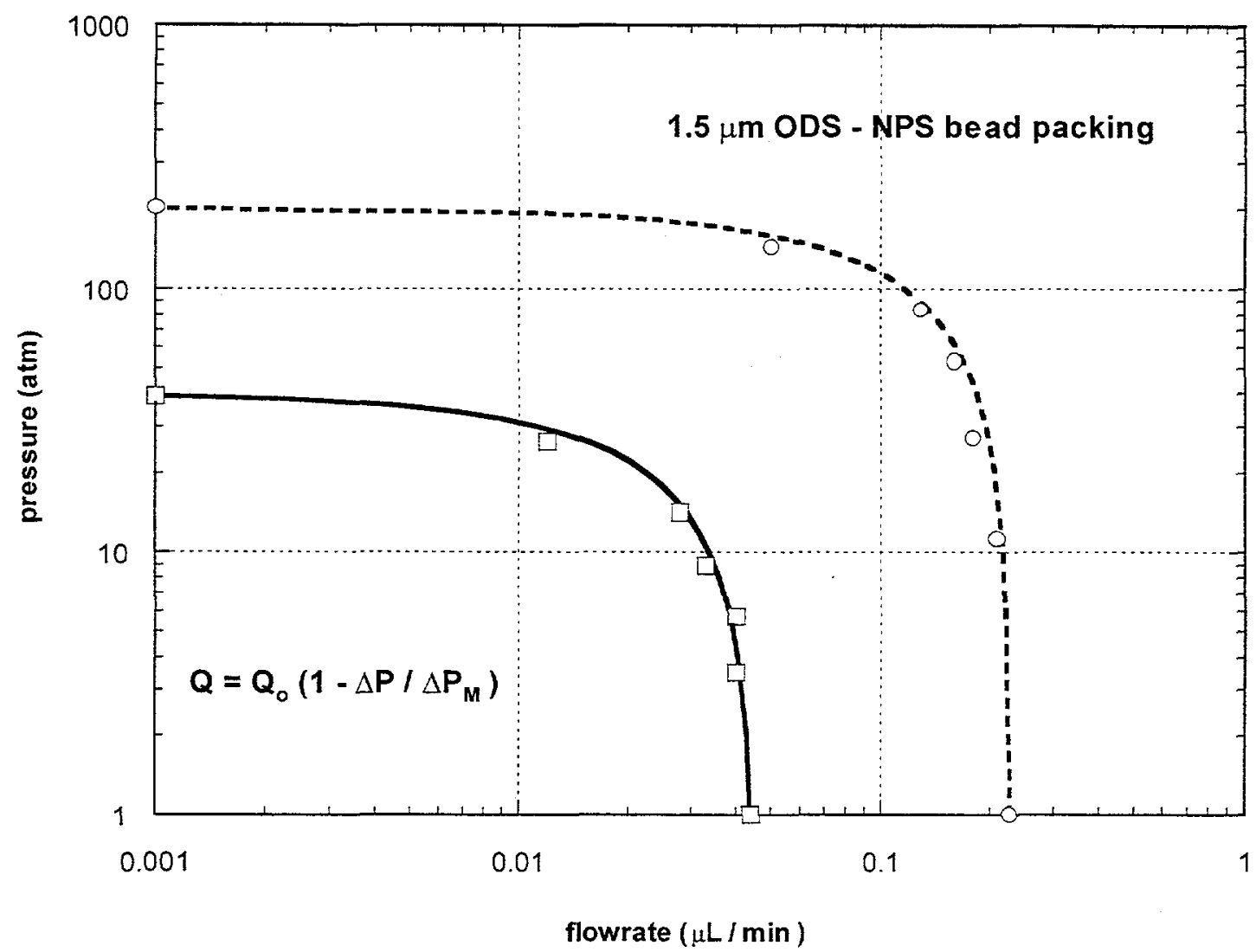

Figure 4. Measured flowrate for operating voltages of 1500 and $6750 \mathrm{~V}$ as a function of back pressure using a packing of 1.5 $\mu \mathrm{m}$ ODS coated NPS beads, 80:20 $\mathrm{ACN}$ :water with $4 \mathrm{mM}$ borate aqueous. Data points plotted on the $\mathrm{x}$ and $\mathrm{y}$ axis indicate the measured flowrate with no applied back pressure and the measured pressure at zero net flow, respectively. The curves are drawn according to equation 9. 


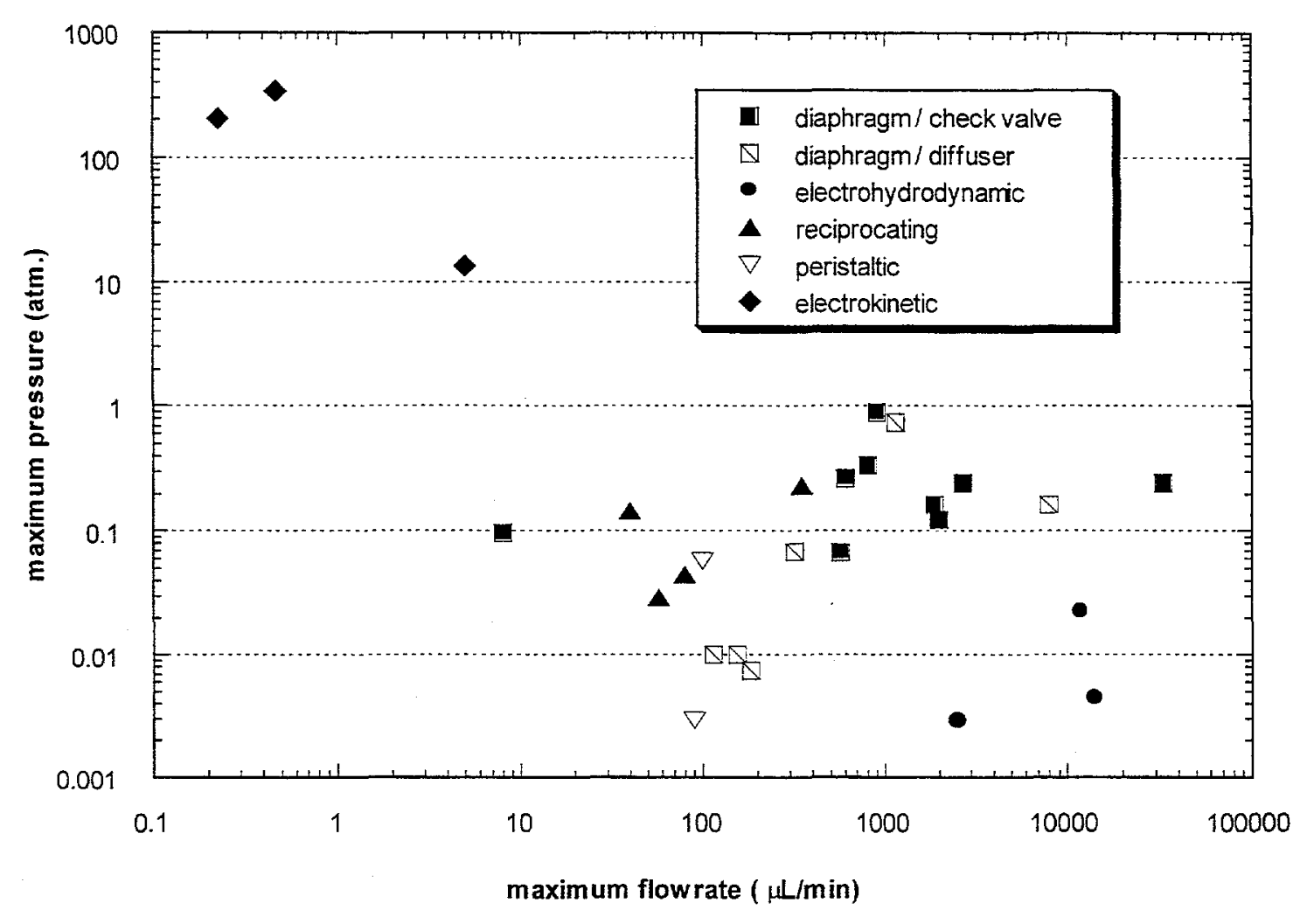

Figure 5. Performance of different types of micropumps. Data points represent reported zero-load flow rates and maximum pressures. 


\section{DISTRIBUTION:}

1. MS 0188

D. Chavez, 4532 for LDRD Office

$25 \quad$ MS 9051

P. H. Paul, 8351

$3 \quad$ MS 9018

Central Technical Files, 8940-2

$1 \quad$ MS 0899

Technical Library, 4916

$1 \quad$ MS 9021

Technical Communications Department, $8815 /$

Technical Library, MS 0899, 4916

1. MS 9021

Technical Communications Department, 8815 For DOE/OSTI

$1 \quad$ MS 0161

Patent and Licensing Office, 11500 